\title{
Results from the First Engineering Run of BOLOCAM and Plans for the Future
}

\author{
P. Mauskopf*, P. Ade*, J. Bock ${ }^{\dagger}$, S. Edgington**, S. Golwala**, A. Goldin ${ }^{*}$, J. \\ Glenn $^{\S}$, D. Haig*, V. Hristov**, B. Knowles ${ }^{\S}$, A. Lange ${ }^{* *}$, H. Nguyen ${ }^{*}$ and B. \\ Rownd ${ }^{\S}$ \\ ${ }^{*}$ Cardiff University - Cardiff - Wales - UK \\ ${ }^{\dagger} J P L$ and Caltech - Pasadena-CA \\ ${ }^{* *}$ Caltech - Pasadena $-C A$ \\ ${ }^{\ddagger} J P L$ - Pasadena - CA \\ ${ }^{\S}$ University of Colorado - Boulder - CO
}

\begin{abstract}
We present results from the engineering run of the BOLOCAM receiver at the Caltech Submillimeter Observatory in May, 2000 and simluations of the sensitivity of the completed BOLOCAM receiver to astrophysical sources. BOLOCAM is a 144 element array of bolometers designed to operate in the millimeter wave atmospheric windows at 150,220 , and $280 \mathrm{GHz}$. These bands are well suited to studies of the Sunyaev-Zel'dovich effect in distant clusters of galaxies as well as measurements of the emission of interstellar dust.
\end{abstract}

\section{INTRODUCTION.}

BOLOCAM is one of a new generation of cameras designed for imaging and surveys at millimeter and sub-millimeter wavelengths. Submillimeter and millimeter wave cameras mounted on the JCMT [1] and on the IRAM 30 meter telescope [2] have begun to perform large area surveys containing sources corresponding to dust emission from distant galaxies (e.g. [3],[4]). At lower frequencies, interferometric arrays have begun to perform surveys of the SunyaevZel'dovich effect in distant clusters [5],[6]. These measurements probe the history of galaxy and cluster formation since the intensity of signals from both dusty galaxies and the SZ effect diminish less rapidly with redshift than optical or X-ray signals. We plan to continue this effort with BOLOCAM observations of large areas of blank sky regions selected to have complementary data at many other wavelengths.

\section{INSTRUMENT}

The combination of several new technologies in BOLOCAM allows new methods of observation that have the potential for low systematic errors and offer sensitivity to signals at a wide variety of angular scales. In BOLOCAM, the detectors and electronics are designed to have no intrinsic excess noise in integrations lasting up to one minute in time. While integrating, the dominant source of noise is from changes in atmospheric emission which must be removed by subtracting the average signal of all of the detectors in the array. This limits BOLOCAM's sensitivity to flux from sources larger than the size of the array on the sky. Because the optics only have to be designed to give diffraction limited performance at a single position of the secondary mirror, the available field of view is maximized and can be significantly larger than the typical chop throw of a secondary mirrow. This also allows the maximum possible number of detectors in the focal plane.

The design of BOLOCAM is described in detail elsewhere [7]. Here, we present a summary of the performance of the system during an engineering run at the Caltech Submillimeter Observatory (CSO) in May, 2000. 


\section{Detectors}

The detectors in BOLOCAM are 'spider-web' type bolometers fabricated as a monolithic array on a three inch silicon wafer. Different configurations of these devices have been designed for use in a variety of experiments including SuZIE [9], BOOMERANG [10], and HERSCHEL (SPIRE) [11]. For the BOLOCAM engineering run, we used a prototype of the 144 element bolometer array that contained a total of 62 working bolometers.

\section{Cryogenics}

For ground-based operation, a base temperature of $300 \mathrm{mK}$ for the bolometers is sufficient to approach photonlimited noise performance. The BOLOCAM array is cooled to a base temperature of $256 \mathrm{mK}$ using a closed cycled adsorption $\mathrm{He}^{3} / \mathrm{He}^{4}$ refrigerator. The fridge contains 3 independent volumes, each consisting of a space for collecting condensed liquid and a cryopump, one charged with $\mathrm{He}^{4}$ and the other two with $\mathrm{He}^{3}$ gas. The $\mathrm{He} 4$ refrigerator is used to condense the gas in the two $\mathrm{He}^{3}$ refrigerators during the cool-down cycle. One of the $\mathrm{He}^{3}$ fridges, the InterCooler (IC) is designed to have a maximal hold time as a function of thermal input by allowing the temperature of the He 3 liquid to be almost unconstrained. This fridge incorporates a long pump tube and heat exchanger that efficiently extracts the heat of enthalpy from the evaporating $\mathrm{He}^{3}$ gas and serves as athermal buffer to the other fridge. In operation it reaches a base temperature of about $400-500 \mathrm{mK}$. The second $\mathrm{He}^{3}$ fridge, the UltraCooler (UC) has a shorter pump tube that is thermally shorted in the middle to the IC cold head to minimize parasitic heat loads and obtain a minimum base temperature.

One advantage of the BOLOCAM fridge is the ability to cycle it without pumping on the main $\mathrm{He}^{4}$ bath. The total fridge cycle takes 1.5 hours and is fully electrical and automated at the telescope. The hold time of the fridge is limited by the hold time of the IC buffer fridge and was measured to be over 24 hours when BOLOCAM was mounted on the CSO (the fridge was cycled once per day and was always still cold at the beginning of the cycle). Details of the design and performance of the fridge are given in [8].

The cryostat is a standard $\mathrm{LHe} / \mathrm{LN}_{2}$ cryostat from Precision Cryogenics. The volume of the $\mathrm{LHe}$ and $\mathrm{LN}_{2}$ tanks are 15 litres each. The consumption of LHe at the CSO during the engineering run was 10 litres per day. We expect to be able to reduce the consumption by at least a factor of 2 in the future.

\section{Electronics}

The readout electronics are designed to give detector noise limited signals in a frequency band from $0.015-20 \mathrm{~Hz}$. The readout circuit is based on the circuit used in the BOOMERANG instrument. This readout scheme has been field tested by BOOMERANG and found to have good performance over the entire frequency band. In adapting the BOOMERANG design for the larger number of bolometers in BOLOCAM, we used surface mount components to minimize the space needed for the electronics. We also changed the first warm preamplifier from an AD624 to INA103 which has a lower white noise specification $(1 \mathrm{nV} / \sqrt{\mathrm{Hz}} \mathrm{vs} .4 \mathrm{nV} / \sqrt{\mathrm{Hz}})$. Laboratory tests of the BOLOCAM electronics after the engineering run showed that the surface mount components used in the BOLOCAM preamplifier boards as well as the INA103 amplifiers have a higher temperature coefficient than the components used in the BOOMERANG electronics. This leads to a large $1 / \mathrm{f}$ noise that is not completely correlated from one amplifier channel to another and therefore cannot be removed. This was observed during the engineering run as an excess noise below $1 \mathrm{~Hz}$ over the detector noise of between a factor of 2-4. Selecting surface mount components with lower temperature coefficient and using the AD624 chips gives back the expected performance.

\section{Optics}

The optical design for BOLOCAM provides an unobstructed 9 arminute field of view diffraction limited at $1 \mathrm{~mm}$ wavelength, the maximum size allowed by apertures in the back structure of the CSO. The reimaging optics and receiver are mounted together in a box attached to the back structure of the telescope near the Cassegrain focus. Two flat mirrors fold and direct the incoming radiation from the telescope onto a $36 \times 36 \mathrm{~cm}$ ellipsoidal mirror that reimages the Cassegrain focus to a point inside the BOLOCAM cryostat and reimages the primary mirror onto a $4 \mathrm{~K}$ aperture 
directly behind the entrance window of the cryostat. This $3.5 \mathrm{~cm}$ diameter aperture is undersized in order to define the illumination pattern of the bolometers on the primary mirror and control spillover. Behind this $4 \mathrm{~K}$ Lyot stop, there is a single lens, $10 \mathrm{~cm}$ in diameter, of high density polyethylene, with a focal length equal to its distance from the Lyot stop. This lens acts to flatten the overall field curvature of the reimaging optics while ensuring that the detector illumination overlaps as much as possible on the primary mirror. A monolithic array of 151 hexagonally packed conical horns with entrance aperture of $5 \mathrm{~mm}$ each is placed at the image plane.

The illumination pattern of the conical horns is larger than the size of the Lyot stop. Therefore, a combination of the horn geometry and the $4 \mathrm{~K}$ cold aperture contribute to the definition of the illumination pattern of the detectors on the primary mirror and on the sky.

The filter bands are determined by a combination of the high pass wave guide cutoff from the section of circular waveguide at the exit aperture of the conical feed horns and an $80 \mathrm{~mm}$ diameter low pass filter placed in front of the horn array. Additional blocking filters are placed at the $4 \mathrm{~K}$ aperture and on the $77 \mathrm{~K}$ shield. An initial test of the filters and horns with five bolometers gave an average optical efficiency for the system of $19 \%$. However, the prototype array used in the engineering run described in this paper had an average optical efficiency of $<7 \%$ mounted in the same configuration using the same horns and filters. The reason for the poor efficiency of the prototype array is most likely due to poor metallization of the absorber on the bolometers.

\section{Scan Strategy}

During the engineering run, all observations were made using drift scans, with the telescope fixed in azimuth and elevation while the earth's rotation caused the source to move across the field of view. This type of scan minimizes sources of environmental signal modulations from moving optical components or sidelobe pickup from the ground. However, it also causes the astrophysical signals to change very slowly and therefore relies on the stability of the detectors and electronics. For a point source observation with a Gaussian beam with FWHM $=40$ ", the $3 \mathrm{~dB}$ point of the signal bandwidth is at a frequency of $80 \mathrm{mHz}$.

\section{OBSERVATIONS}

BOLOCAM was first mounted on the CSO for five nights from May $18-22,2000$. The first two nights were devoted to setup and commissioning tests while the last three nights were devoted to test observations. Data was obtained in observations of Uranus, 3C273, W58, G34.3, DR21, and Abell 1835, all in drift scan mode. Here we present preliminary maps of Uranus, DR21, and Abell 1835.

\section{DATA ANALYSIS}

\section{Merging}

The signals from the BOLOCAM bolometers are sampled at $50 \mathrm{~Hz}$ by a PC-based data acquisition system. At the same time, the pointing data from the telescope computer are written to a different file at a rate of $100 \mathrm{~Hz}$. In both data streams there are common TTL signals used for communication between systems on BOLOCAM and the telescope computer and for synchronization of the data streams. These TTL signals include signals from the telescope that switch from high to low when the telescope changes from tracking mode to drifting mode. These signals are the primary ones used to synchronize the data streams and are also used to mask out data from the cleaning and map making processes when the telescope is slewing.

Both the raw bolometer data files and the telescope data files are written to disk once per minute. A merging routine combines these files once every several minutes into a single time stream that contains all of the receiver and telescope data. 


\section{Cleaning}

After the data has been combined into a single file, we perform a deconvolution of the electronics transfer functions and remove common mode noise in the detectors which is dominated by common-mode sky temperature fluctuations. To do this, we first separate the data in the time stream into individual drift scans using the TTL signals from the telescope. Then, we remove spikes in the data and deconvolve scan by scan. The bolometer time constants are comparable to the $20 \mathrm{~Hz}$ bandwidth of the readout electronics, so cosmic ray events only last a few samples and are easily removed. We remove an average of $2 \%$ of the data in a given bolometer.

After despiking, we remove the common mode signals from across the array. The output voltage from each detector can be described by:

$$
V_{i}(t)=\frac{\mathrm{dR}_{\mathrm{i}}}{\mathrm{dP}} *\left(F\left(\theta_{i}(t)\right)+\mathrm{A}(\mathrm{t})\right)+\delta \mathrm{I}_{\text {bias }}(\mathrm{t}) \mathrm{R}_{0}+\frac{\delta \mathrm{G}_{\mathrm{i}}(\mathrm{t})}{\mathrm{G}} \mathrm{I}_{\text {bias }} \mathrm{R}_{0}+\mathrm{e}_{\mathrm{i}}(\mathrm{t})
$$

where $F(\theta)$ is the flux on the sky as a function of angle, $A(t)$ is the common mode emission of the atmosphere + telescope as a function of time, $e_{i}$ is the detector noise, $\mathrm{R}_{\mathrm{i}}$ is the bolometer resistance, $\mathrm{G}$ is the electronics gain, and $\mathrm{I}_{\text {bias }}$ is the common bias current. The five terms in equation $\mathrm{xx}$ are: $\left.\mathrm{i}\right)$ sky signal $\propto F\left(\theta_{i}(t)\right)$, ii) sky noise $\propto \mathrm{A}(\mathrm{t})$, iii) bias noise $\propto \delta \mathrm{I}_{\text {bias }}(\mathrm{t})$, iv) electronics gain noise $\propto \frac{\delta \mathrm{G}_{\mathrm{i}}(\mathrm{t})}{\mathrm{G}}$ and, v) detector noise. Terms (ii) and (iii) are common mode and can be removed by cleaning.

In addition, to these components, we find a component of the signal that is correlated within each hextant or group of 24 channels all going through the same preamplifier boards, cryogenics cables, JFET boards, etc., but not correlated from one hextant to another. This component of noise is most likely from gain variations in the preamplifier boards due to temperature variations. To remove it, we make an average of the signals within each hextant and remove that from the individual bolometers within the hextant instead of removing the average over the entire array. The resulting cleaned data has about a factor of 1.5 less noise at low frequencies. Finally, we also either remove a 2 nd order polynomial from each scan or reapply a high pass filter to the time ordered data in order to reduce the effect of remaining $1 / \mathrm{f}$ noise in the time stream.
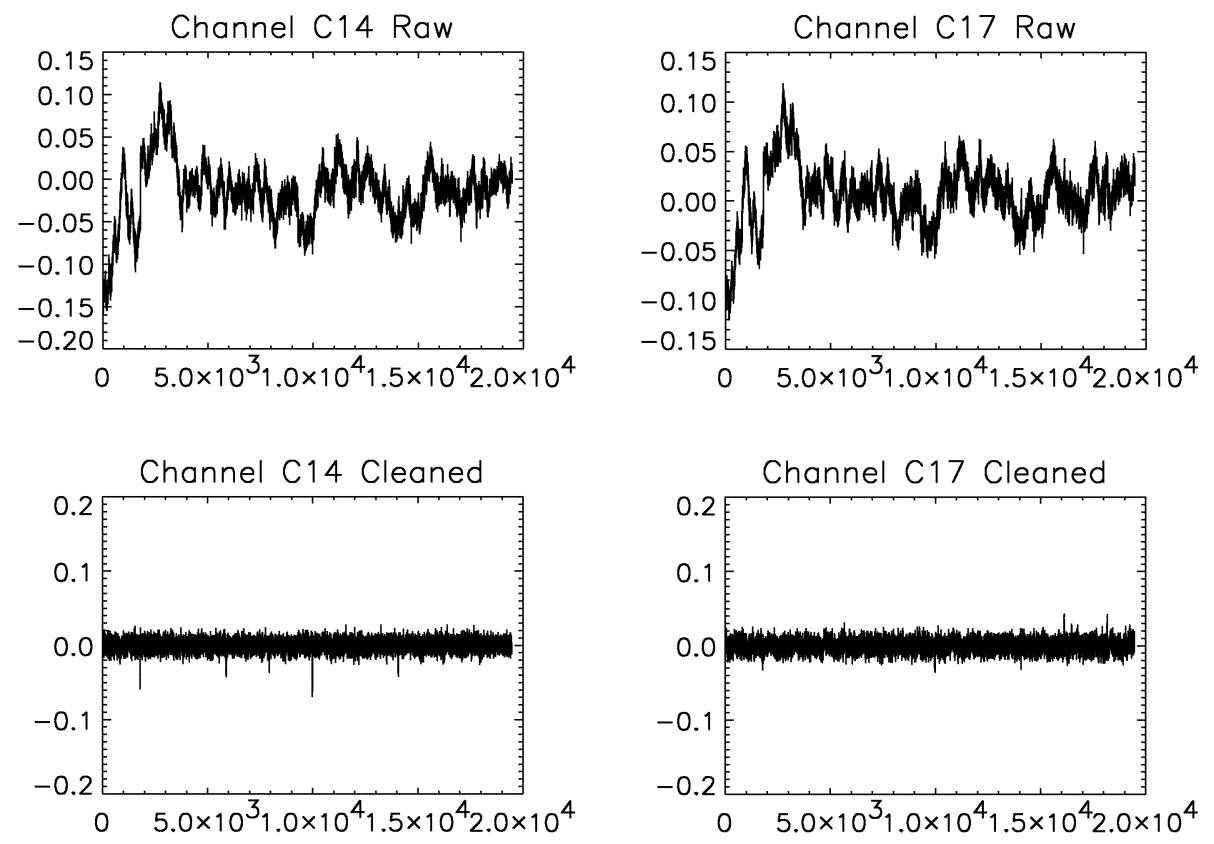

FIGURE 1. BOLOCAM data before and after cleaning

In observations where there is a bright source present, the removal of a polynomial or the application of a high pass filter creates shadows, or regions of "negative flux" around the source. In order to avoid this, we allow the option of running a source removal module that masks out all data within 2 beam FWHM around specified sources in the subsequent cleaning and low frequency noise removal modules. This eliminates the shoadowing effect. 


\section{Mapping}

After the data has been cleaned, we make binned maps on the sky from the time stream, using the pointing data from the telescope and the data from the rotator. We first calibrate the raw time stream of each detector using the individual detector responsivities measured in observations of Uranus. We then average data from all of the detectors in each bin, weighted by the noise integrated in a band from $100-200 \mathrm{mHz}$. We compute a weighted mean and variance from the data in each bin.

\section{RESULTS}

Figure 2 shows a map of one observation of the planet Uranus. The map is made from a total of 114000 samples from each of 62 bolometers, corresponding to a total observation time of 2280 seconds. Of this total observing time, a total of 66373 samples from each bolometer are actually used in the map, an observing efficiency of 58\%. The map is pixellized in $5 " \times 5$ " pixels and covers a total area of $200 \mathrm{sq}$. arcmins. The map was made in a raster scan mode, the telescope stepping in declination between each drift scan with a step size of 5". The rotator was running during the observation to maintain the same array rotation with respect to parallactic angle at all times.

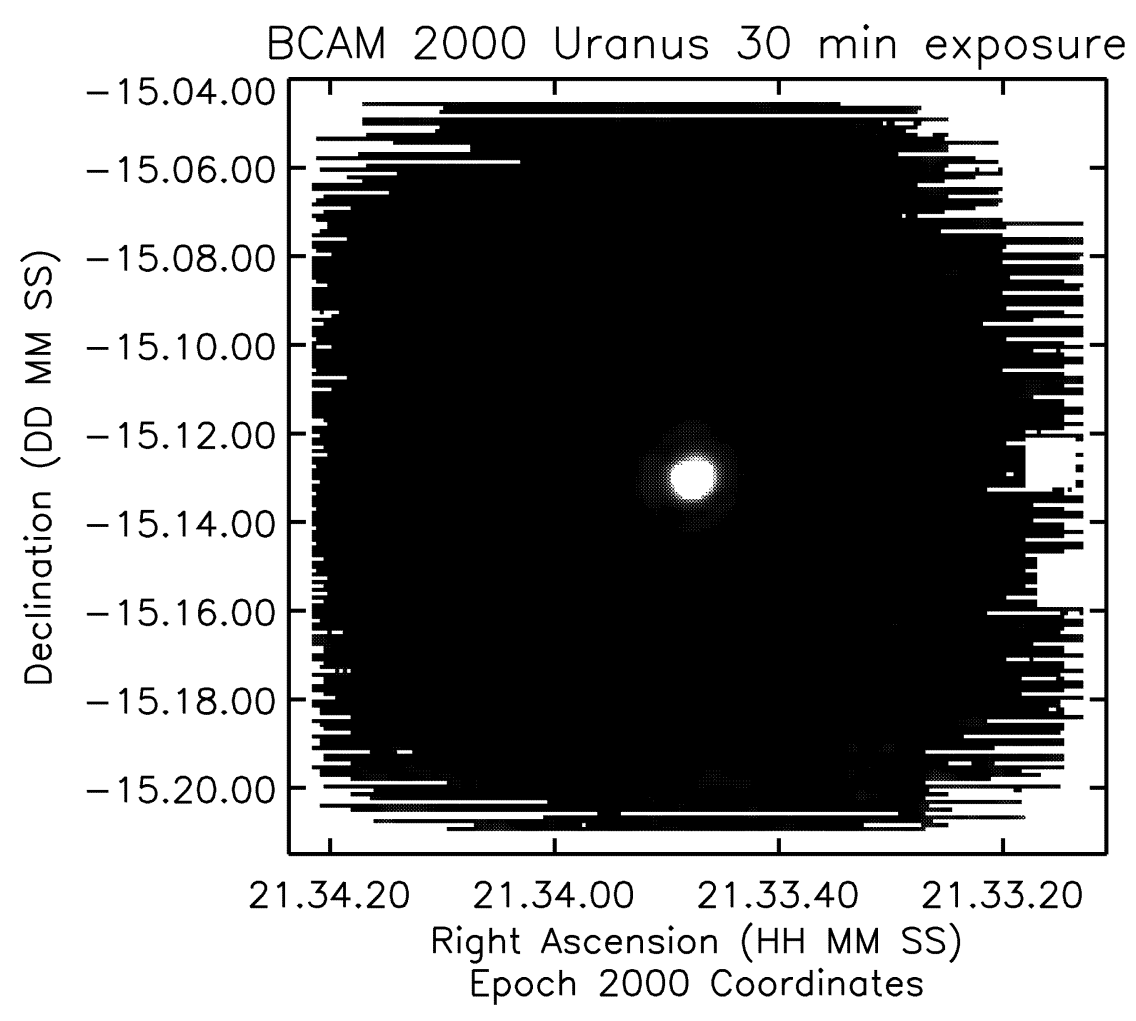

FIGURE 2. Map from one 30 minute observation of Uranus

From the map we derive the following average beam parameters: solid angle $=0.43 \mathrm{sq}$. arcmins, beam $\mathrm{FWHM}=38$ " in agreement with the expected values from the optical design. The flux from Uranus in the BOLOCAM $1.4 \mathrm{~mm}$ band is approximately $30 \mathrm{Jy}$ [12]. There is an Airy ring clearly visible in the map and in the radial profile plot (Figure 3) with an amplitude equal to $1 \%$ of the peak amplitude. In addition, there is a negative going feature at a radius of about $5 \sigma$ from the beam center with an amplitude of $<0.3 \%$ of the peak. The error per 5 "x 5 " pixel in the center of the map 
is approximately $80 \mathrm{mJy}$, corresponding to an error of $12 \mathrm{mJy} / \mathrm{beam}$, and a signal to noise ratio in the central 5 " $\times 5$ " pixel of almost 500 .

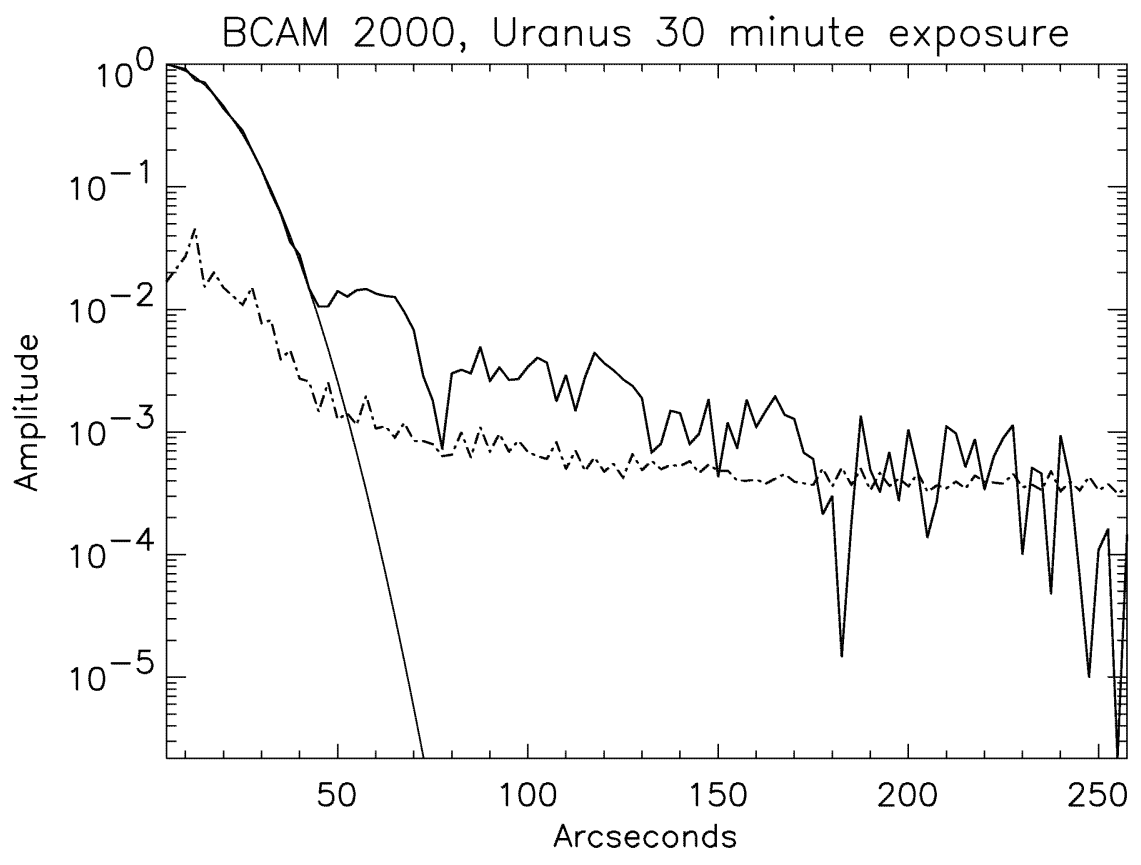

FIGURE 3. Plot of radially averaged flux from Uranus map. The solid line is the raw data overlaid with a Gaussian profile with FWHM $=38$ ". The dashed line is the error measured from the variance of the pixels in the radially averaged bin.

\section{A1835}

Figure 4 shows a map of the region around the cluster Abell $1835(\mathrm{z}=0.2523)$. This cluster is one of the most luminous X-ray emitting clusters in the sky with a total X-ray luminosity of $3.8 \times 10^{45} \mathrm{erg} / \mathrm{s}$ [15], a core radius of $\theta_{\text {core }}=0.22^{\prime}$ for a King model with $\beta=0.66$. It is one of the most studied clusters of galaxies in the wavelength region from the radio through the submillimeter. It has a strong SZ thermal signal with a measured peak y-parameter of $4.6 \times 10^{-4}$ [14] and also has a strong central radio source with a flux of $3 \mathrm{mJy}$ at $30 \mathrm{GHz}$ [16] and a central sub-mm dust source with a central flux of $20 \mathrm{mJy}$ at $450 \mathrm{um}$ [13]. We integrated on this region for a total of 3 hours during the last night (May 22) of the BOLOCAM engineering run.

The map is approximately $10^{\prime} \times 16^{\prime}$ in size, pixellized in $5 " \times 5$ " pixels and has been smoothed with a Gaussian smoothing function with a FWHM $=40 "$ " The observations were made in drift scan mode with three declination steps of 5" each in order to evenly fill in the sampling an the sky. The map is made from a total of 564000 samples of which 339685 are actually used in the final map or $60 \%$ observing efficiency. The error per pixel in the smoothed map is $5 \mathrm{mJy}=100 \mu \mathrm{K}_{\mathrm{R}-\mathrm{J}}=300 \mu \mathrm{K}_{\mathrm{CMB}}$. We perform a fit of the unsmoothed map to the cluster profile smoothed to the BOLOCAM beam size and find a most likely value of the peak amplitude of the SZ signal at $1.4 \mathrm{~mm}$ of $-170 \pm 340 \mu \mathrm{K}_{\mathrm{CMB}}$, which corresponds to a flux in a BOLOCAM beam centered on the cluster of $-1.5 \pm 3 \mathrm{mJy}$.

\section{SENSITIVITY}

We calculate our sensitivity in several different ways. We calculate the sensitivity of each detector to sky signal as a function of frequency.

$$
S(\mathrm{mJy} / \sqrt{\mathrm{Hz}})=\operatorname{PSD}(\mathrm{V} / \sqrt{\mathrm{Hz}}) \mathrm{Jy}_{\text {Uranus }} / \mathrm{V}_{\text {Uranus }}
$$




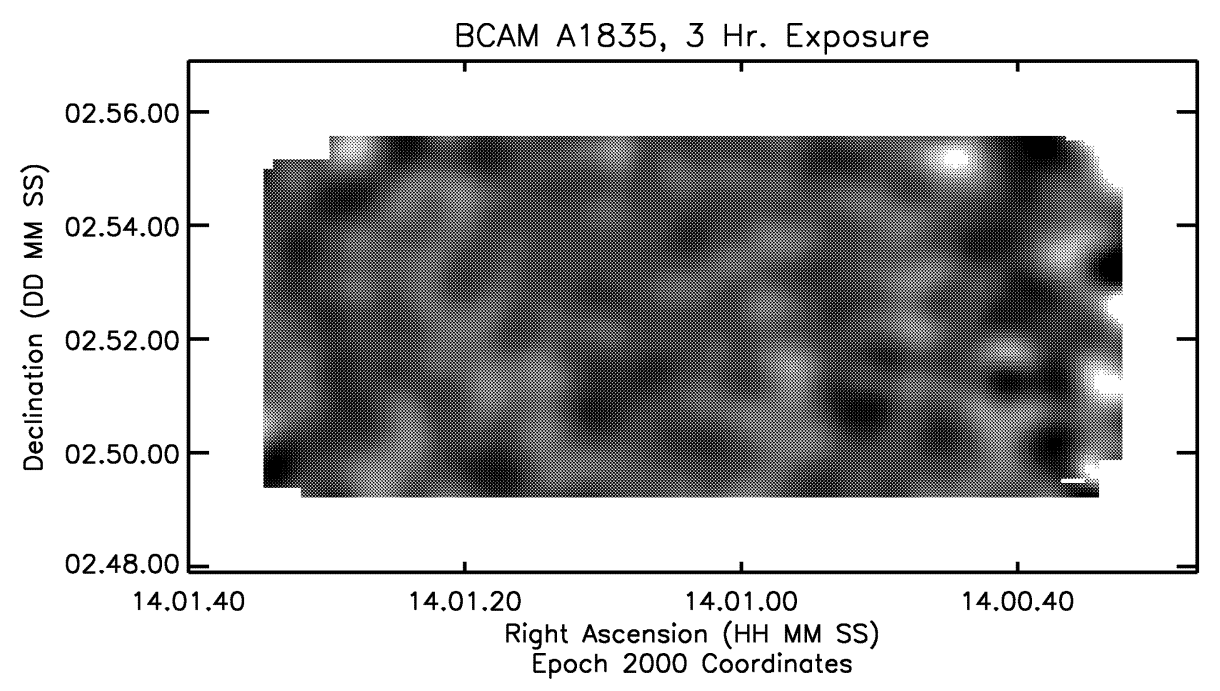

FIGURE 4. Map of the region of sky near the cluster Abell 1835

Where the PSDs are calculated from the cleaned data and calibrated by the peak signal from each bolometer on observations of Uranus. Figure 5 shows a plot of the sensitivity of a good bolometer vs. frequency. As described earlier, there is excess noise from the prototype electronics at low frequencies that remains after cleaning and therefore is not correlated from bolometer to bolometer. The white noise of the detector is about $50 \mathrm{mJy} / \sqrt{\mathrm{Hz}}$ while the noise at the effective frequency of the drift scan observations is $100-200 \mathrm{mJy} / \sqrt{\mathrm{Hz}}$. This is a factor of $4-8$ times worse than the optimum sensitivity for a single pixel. The factors contributing to this loss in sensitivity are:

1. Optical Efficiency of prototype wafer: $2-3$

2. Excess 1/f noise in electronics: $2-4$

We also calculate the overall sensitivity of the array from the noise in the maps in terms of a mapping speed. Mapping speed is defined as the solid angle that it is possible to map down to a given noise level in a given amount of time (ref. Glenn). This is given by:

$$
\mathrm{M}\left(\operatorname{arcmin}^{2} / \mathrm{mJy}^{2} / \mathrm{Hr}\right)=3600(\mathrm{sec} / \mathrm{Hr}) \mathrm{N}_{\text {det }} \varepsilon_{\text {int }} \Omega_{\text {beam }}\left(\operatorname{arcmin}^{2}\right) / \mathrm{S}_{\text {det }}^{2}(\mathrm{mJy}, \sqrt{\mathrm{sec}})
$$

where $\varepsilon_{i n t}$ is the efficiency of integration time. For the Abell 1835 observation, we find an effective mapping speed of the array of:

$$
\mathrm{M}=0.7 \operatorname{arcmin}^{2} / \mathrm{mJy}^{2} / \mathrm{Hr}
$$

\section{CHANGES}

Since the May, 2000 observing run, we have made several changes to the instrument. First, we have fabricated a new wafer which has over 100 good bolometers for which have measured optical efficiencies about 2.5 times higher than the detectors in the prototype array. Second, we have modified the readout electronics and eliminated the excess $1 / \mathrm{f}$ noise so that the readouts are now consistent with white noise down to frequencies $<30 \mathrm{mHz}$. Finally, we have changed the filters and horn array to observe at a wavelength of $2.1 \mathrm{~mm}$ rather than $1.4 \mathrm{~mm}$. Currently we are in the final stages of preparation for a second engineering run at the CSO in December, 2001. Taking these improvements into account, we expect to achieve a mapping speed of:

$$
M \simeq 100 \operatorname{arcmin}^{2} / \mathrm{mJy}^{2} / \mathrm{Hr}
$$

or

$$
M \simeq 100 \operatorname{arcmin}^{2} /(35 \mu \mathrm{K})^{2} / \mathrm{Hr}
$$




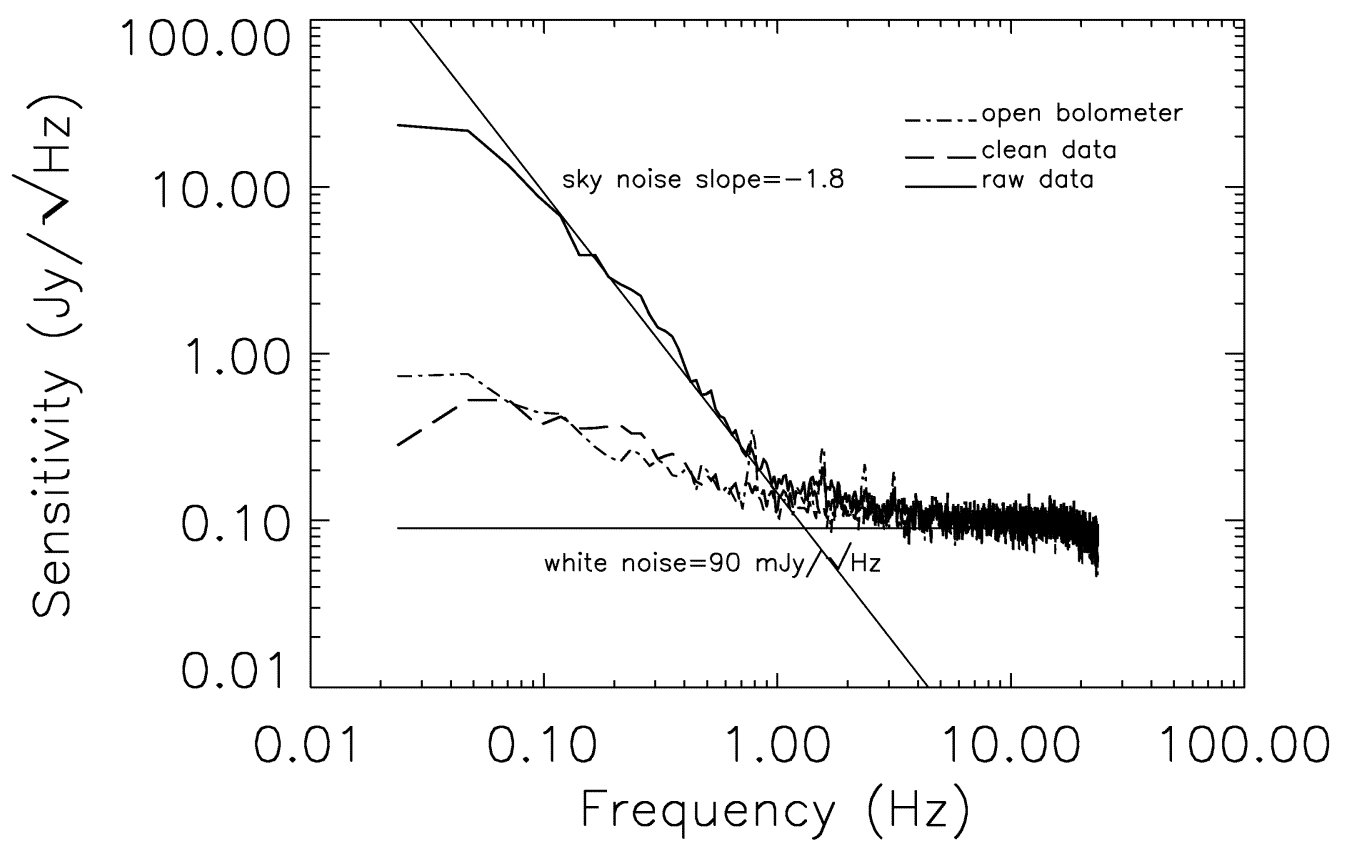

FIGURE 5. PSDs of BOLOCAM channel before and after cleaning, with a PSD of an open channel showing only electronics noise

including observing overheads during the upcoming run. With this mapping speed, we can map over one square degree to an error of less than $20 \mu \mathrm{K}$ per beam in 50 hours of observation and to $12 \mu \mathrm{K}$ per beam in 100 hours.

\section{SIMULATIONS}

We have used the estimated mapping speeds above to perform simulations of observations with BOLOCAM during the observing run this December. Figure 6 shows maps of a $1^{\circ} \times 1^{\circ}$ degree region of sky with and without detector noise added assuming 100 hours of integration time. The sky signal is a combination of primary and secondary CMB anisotropies. The primary anisotropy signal is generated from a $\Lambda$-CDM model that fits the current CMB power spectrum data at lower multipoles. The secondary SZ anisotropies are taken from hydrodynamical simulations for flat $\Lambda$ cosmology. The overall rms from the sky is $20 \mu \mathrm{K}$ of which the SZ signal contributes an rms of $12.5 \mu \mathrm{K}$ and the primary CMB anisotropy on angular scales from the BOLOCAM beam size of 1' to the 8' size of the array contributes an rms of $15.5 \mu \mathrm{K}$. The noise rms in 1' pixels is $12 \mu \mathrm{K}$. There are 8 clusters in the map with a signal to noise ratio greater than 4.

\section{CONCLUSIONS}

We have presented some preliminary results from the first run of the BOLOCAM instrument at the CSO in May, 2000. During 5 nights of observation, the instrument was successfully integrated with the telescope and observations were made of various galactic and extragalactic sources. Since this engineering run, a new wafer of bolometers for the focal plane has been produced with over 100 working detectors and the nominal optical coupling to incoming radiation, which is a factor of 2-3 improvement in coupling over the engineering wafer. In addition, the readout electronics have been upgraded to eliminate excess $1 / \mathrm{f}$ noise present during the engineering run. Finally, a full software pipeline has been developed and used to analyze the data taken from the engineering run. These improvements will be tested in detail during the next engineering run of BOLOCAM on the CSO this December. 

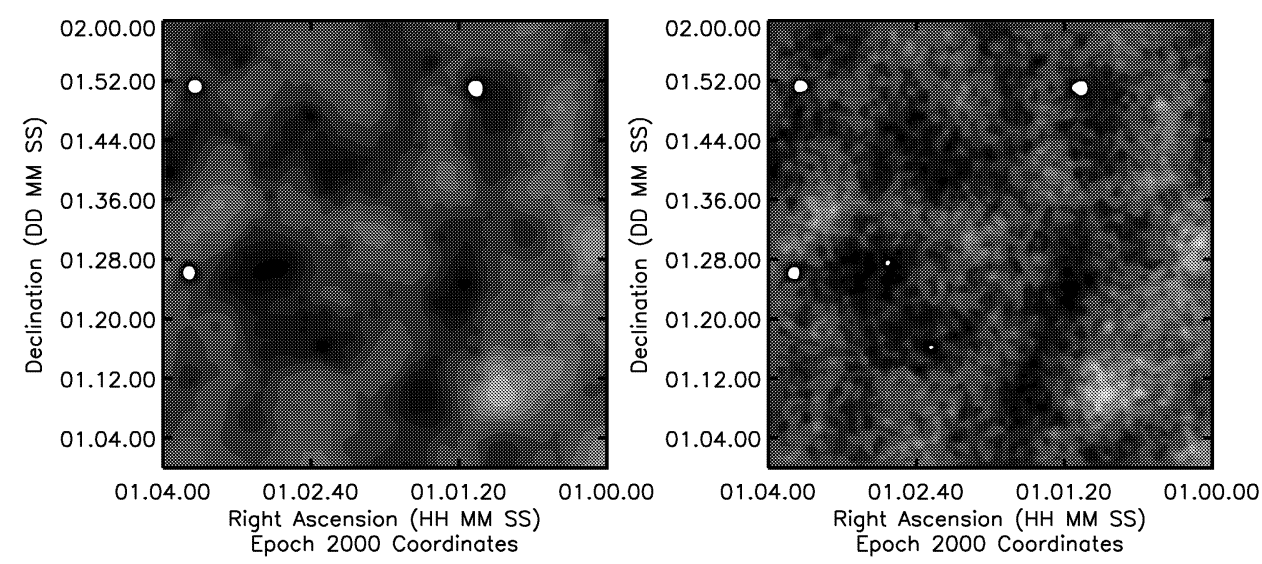

FIGURE 6. Simulation of $1^{\circ} \times 1^{\circ}$ field at $150 \mathrm{GHz}$ with and without simulated instrument noise

\section{ACKNOWLEDGMENTS}

This activity is being supported by the US Agencies NASA and NSF and by PPARC, and by the Leverhulme Trust in the UK.

\section{REFERENCES}

1. Holland, W., et al., 1999, MNRAS, 303, 659

2. Kreysa, E., et al., 1998, Proc. SPIE Vol. 3357, p. 319-325, Advanced Technology MMW, Radio, and Terahertz Telescopes, Thomas G. Phillips; Ed.

3. Eales, S., et al., 2000, ApJ, 120, 2244-2268

4. Bertoldi, F., 2000, A\&A, 360, 92-98

5. Joy, Marshall, et al., 2001, ApJ, 551, L1-L4

6. Jones, M., et al., 2001, MNRAS (submitted), astro-ph/0103046

7. Glenn, J., et al., 1998, Proc. SPIE Vol. 3357, p. 326-334,

8. Bhatia, R., et al., 2000, Cryogenics, 40, 685-691

9. Mauskopf, P. D., et al., 1997, Appl. Opt., 36, p. 765

10. , Bock, J., et al., "Infrared Bolometers With Silicon Nitride Micromesh Absorbers", Proceedings of "Submillimetre and Far-Infrared Space Instrumentation", 30th ESLAB Symposium 24-26 September 1996, ESTEC, Noordwijk, Netherlands.

11. Bock, J., et al., 1998, Proc. SPIE Vol. 3357, p. 297-304, Advanced Technology MMW, Radio, and Terahertz Telescopes,

Thomas G. Phillips; Ed.

12. Griffin, M. J. and Orton, G. S., 1993, Icarus, 105, 537

13. Edge A., et al., 1999,MNRAS, 306, 599-606.

14. Mauskopf P., et al., 2000, Ap.J., 538, 506-516.

15. Allen, S., et al. 1996, MNRAS, 283, 263-281.

16. Cooray A., 1999, New A., 4, 377-388. Advanced Technology MMW, Radio, and Terahertz Telescopes, Thomas G. Phillips; Ed. 